\section{STENTLESS PORCINE AORTIC ROOT: VALVE OF CHOICE FOR THE ELDERLY PATIENT WITH SMALL AORTIC ROOT?}

The Medtronic Freestyle bioprosthesis is a stentless porcine aortic root cross-linked in dilute glutaraldehyde solution with stress-free fixation for the valve leaflets. It has been treated by a process in which amino oleic acid is used to reduce the potential for calcification. As a complete aortic root, it has the same versatility as the aortic homograft but has the advantage that it is readily available in all sizes to the implanting surgeon. Between January 1993 and May 1994, we implanted 64 Freestyle bioprostheses as aortic valve replacements using a freehand technique; 5 size $19 \mathrm{~mm}, 15$ size $21 \mathrm{~mm}, 16$ size $23 \mathrm{~mm}, 13$ size $25 \mathrm{~mm}$, and 15 size $27 \mathrm{~mm}$ valves were used. There were 35 men and the mean age was 75.7 years ( 64 to 84 years). The operative mortality was $3.1 \%(2 / 64)$. Echocardiograms at the time of discharge revealed mean aortic valve gradients ranging from $18.2 \mathrm{~mm} \mathrm{Hg}$ for $19 \mathrm{~mm}$ to 10.3 $\mathrm{mm} \mathrm{Hg}$ for $27 \mathrm{~mm}$ valves. Effective orifice areas ranged from $1.0 \mathrm{~cm}^{2}$ for $19 \mathrm{~mm}$ to $2.0 \mathrm{~cm}^{2}$ for $27 \mathrm{~mm}$ valves. No patient had more than trace aortic insufficiency. Our early experience with this new stentless bioprosthesis shows it to have excellent hemodynamics especially in the smaller valve sizes. Using this valve in patients who have a small aortic root and require a tissue valve avoids the need for aortic root enlargement procedures. (J THORAC CARDIOvaSC SURG 1995;109:871-6)

Colleen F. Sintek, MD, Alden D. Fletcher, MD, PhD, and Siavosh Khonsari, MD, Los Angeles, Calif.
$\mathrm{D}$ espite more than 30 years of research and clinical applications, the pursuit for the ideal heart valve substitute continues. In the aortic position, the pulmonary autograft may come close to meeting all of the criteria for a perfect replacement valve; however, the operation does require prosthetic replacement of the pulmonary valve and right ventricular outflow tract. Certainly the mechanical valves with their requirement for anticoagulation and thromboembolic complications are far from ideal and the stented bioprostheses have inferior hemodynamics, especially in the smaller valve sizes. ${ }^{1}$ Homograft valves have been relatively durable and free of thromboembolic problems; however, in the United States their availability is limited. The stentless porcine aortic root appears to be a good alternative to the aortic homograft in terms of its hemodynamics. Like the homograft, the porcine aortic

From the Regional Department of Cardiac Surgery, Kaiser Permanente Medical Center, Los Angeles, Calif.

Read at the Twentieth Annual Meeting of The Western Thoracic Surgical Association, Olympic Valley, Calif., June 22-25, 1994.

Address for reprints: Colleen F. Sintek, MD, Kaiser Permanente Medical Center, 1526 N. Edgemont St., Los Angeles, CA 90027.

Copyright $(\mathcal{C} 1995$ by Mosby-Year Book, Inc.

$0022-5223 / 95 \$ 3.00+0 \quad \mathbf{1 2 / 6 / 6 2 3 6 8}$ root has no stent or sewing ring to obstruct forward flow. It is also believed that the nonstented porcine valve offers the potential for increased durability because of the elimination of the stent. Maintaining the structure of the native coronary sinuses may decrease the mechanical stress on the leaflets by dissipation of energy to the aortic root during closure at high back pressures.

\section{Patients and methods}

The device. The Freestyle aortic root is manufactured by Medtronic Heart Valves, Irvine, California. The leaflets are maintained at zero differential pressure during the glutaraldehyde fixation process. The aortic wall is fixed at $40 \mathrm{~mm} \mathrm{Hg}$ pressure to control the shrinkage of the wall and to maintain the natural symmetry of the commissure attachments to the aortic wall, which minimizes the potential for misalignment during implantation. The inflow aspect of the valve is covered with a single sheet of Dacron polyester, which extends over the myocardium to provide strength to this portion of the bioprosthesis. The right and left coronary arteries are ligated with 5 to $6 \mathrm{~mm}$ remnants that can be used in reimplantation of the patient's coronary arteries when the Freestyle valve is used for root replacement. The Freestyle valve is treated with an $\alpha$-amino derivative of oleic acid, which has been shown in animal studies to mitigate leaflet calcification. Because calcification of valve leaflets is a major cause for failure of porcine bioprostheses, ${ }^{2,3}$ this antimineralization treatment may lead to increased durability of the Freestyle aortic root. 
Table I. Patient demographics and clinical parameters

\begin{tabular}{lcc}
\hline & Mean & Range \\
\hline Total patients & 64 & \\
Male gender & 35 & \\
Age (yr) & 75.7 & $64-84$ \\
AS & 25 & \\
AI & 4 & \\
Combined AS and AI & 35 & \\
Redo sternotomy & 10 & \\
Concomitant CABG & 26 & \\
Average crossclamp time (min) & & \\
$\quad$ Isolated AVR & 89.3 & $73-112$ \\
$\quad$ With CABG & 111.5 & $75-141$ \\
Mean postop. length of stay (days) & 12.7 & $6-48$ \\
\hline AS, Aortic stenosis; $A I$, aortic insufficiency; $C A B G$, coronary artery bypass \\
grafting; $A V R$, aortic valve replacement.
\end{tabular}

Patients. Our institution is one of the study centers in the United States for the Medtronic Freestyle aortic root bioprosthesis. The clinical study is being conducted at multiple centers with all sites using a common investigational plan. Before initiation of the use of the Freestyle valve at our hospital, the study design was approved by our institution's investigational review board. All patients believed to be candidates for bioprosthetic aortic valve replacement were considered for the Freestyle valve. Patients requiring multiple valve procedures were excluded. Written informed consent was obtained from each patient before the operation.

Surgical technique. Surgical technique consisted of standard cardiopulmonary bypass with a single dualstaged venous cannula. Myocardial protection was achieved with moderate systemic hypothermia with antegrade and retrograde cold blood cardioplegia. All patients underwent implantation of the Freestyle bioprosthesis as an aortic valve replacement by means of a freehand technique similar to that used by many for valve replacement with a homograft. An oblique aortotomy was made extending into the noncoronary sinus. The appropriate valve size was selected after the native aortic valve had been excised and the anulus debrided. A deliberate effort was made to avoid undersizing the valve. In fact, the valve usually was oversized slightly on the basis of the patient's anulus size. We constructed the proximal suture line with interrupted 4-0 Ti-Cron sutures (Davis W. Geck, Danbury, Conn.) in a simple fashion, placing these stitches in the valve "anulus" first beneath the nadir of each sinus, placing the sutures approximately $2 \mathrm{~mm}$ apart, and picking up ventricular muscle and subaortic curtain to form a circle of stitches in a single plane. These sutures were brought up through the Dacron skirt of the Freestyle valve, which was then lowered into place, and the sutures were secured. The left and right coronary sinus portions of the Freestyle valve were removed, leaving a 3 to $4 \mathrm{~mm}$ scalloped rim of tissue except over the uppermost aspect of the commissures, where the rim was 5 to $6 \mathrm{~mm}$. The noncoronary sinus portion of the Freestyle valve was left intact. Great care was taken to align the commissures of the Freestyle valve at 120 degrees and to resuspend them at least 2 to $3 \mathrm{~mm}$ above the native commissures. This was accomplished with pledget-supported 4-0 Prolene sutures (Ethicon, Inc., Somerville, N.J.), which were left untied. The distal suture line was completed with running 4-0 Prolene suture, attaching the scalloped portions of the left and right coronary sinuses beneath the patient's coronary ostia and attaching the retained noncoronary portion of the Freestyle valve to the patient's aortic wall. The pledget-supported commissure sutures were then secured.

Study methods. All patients underwent preoperative echocardiograms, intraoperative transesophageal echocardiographic evaluation of the left ventricular outflow tract and aortic valve function, as well as a transthoracic echocardiogram at the time of hospital discharge, 3 to 6 months after the operation, and again 1 year after hospital discharge. From the echocardiogram, mean aortic valve gradient, cardiac output, effective orifice area (EOA), and degree of aortic insufficiency were determined. A few selected patients with small valve sizes underwent cardiac catheterization before hospital discharge. Standard laboratory values, including red blood cell count, leukocyte count, hemoglobin concentration, reticulocyte count, and serum lactic dehydrogenase, serum alkaline phosphatase, haptoglobin, and serum creatinine concentrations were obtained at the same time intervals as the echocardiograms. Operative and long-term mortality data were collected, as well as in-hospital morbidity and complications occurring during the follow-up period.

\section{Results}

Patient data. Between January 1993 and May 1994, 64 patients underwent implantation of Freestyle bioprostheses: 5 size $19 \mathrm{~mm}, 15$ size $21 \mathrm{~mm}, 16$ size $23 \mathrm{~mm}, 13$ size $25 \mathrm{~mm}$, and 15 size $27 \mathrm{~mm}$ valves were implanted. Patient demographics and clinical parameters are listed in Table I.

Operative mortality and morbidity. The 30-day operative mortality was $3.1 \%$ (two patients) (Table II). One in-hospital death occurred on the sixth postoperative day in a patient being readied for hospital discharge. This death occurred suddenly and was probably related to an arrhythmia. Autopsy revealed coronary atherosclerosis with calcified plaques but no evidence of myocardial infarction, and the Freestyle valve appeared to be functioning normally. One patient died on postoperative day 14 after hospital discharge. She was readmitted to another hospital and treated for bilateral pleural effusions with diuretics. She subsequently became hypotensive and died. Postmortem examination revealed bilateral adrenal hemorrhage and acute splenitis. No evidence of purulence was noted either in the chest cavity or on the valve itself, which appeared normal.

Right hemiparesis developed on the second postoperative day in one patient, and transient ischemic 
Table II. Operative results

\begin{tabular}{lcc}
\hline & \multicolumn{2}{c}{ Patients } \\
\cline { 2 - 3 } & No. & $\%$ \\
\hline Thirty-day mortality & 2 & 3.1 \\
Stroke & 1 & 1.6 \\
Transient ischemic attack & 2 & 3.1 \\
Mediastinitis & 2 & 3.1 \\
Gastrointestinal bleeding & 1 & 1.6 \\
Pacemaker & 2 & 3.1 \\
\hline
\end{tabular}

Table III. Hemodynamic results determined by echocardiographic evaluation at the time of discharge (60 patients)

\begin{tabular}{ccc}
\hline Valve size & Gradient $(\mathrm{mm} \mathrm{Hg})$ & EOA $\left(\mathrm{cm}^{2}\right)$ \\
\hline $19 \mathrm{~mm}$ & $18.2(9.0-23.0)$ & $1.0(0.8-1.3)$ \\
$21 \mathrm{~mm}$ & $13.3(5.7-25.3)$ & $1.3(1.0-2.0)$ \\
$23 \mathrm{~mm}$ & $12.2(4.3-19.3)$ & $1.4(1.0-1.7)$ \\
$25 \mathrm{~mm}$ & $9.8(3.2-13.8)$ & $1.7(1.3-2.4)$ \\
$27 \mathrm{~mm}$ & $10.3(2.8-21.8)$ & $2.0(1.1-3.5)$
\end{tabular}

Gradient, Mean aortic valve gradient; $E O A$, effective orifice area; range is given in parenthesis.

attacks occurred on the second and fourth postoperative days, respectively, in two patients. In all three patients results of computed tomographic brain scans were within normal limits. All of these neurologic events occurred early in our series when we were not routinely administering anticoagulants after implantation of the Freestyle bioprosthesis. Subsequent to these events all patients have been maintained on low-dose anticoagulation with warfarin sodium, maintaining an international normalized ratio of 2.0 to 2.5 for the first 3 months after the operation. Since implementing this protocol, we have had no further perioperative neurologic events. No patient has had an intraoperative neurologic injury.

Two patients required surgical intervention for mediastinitis. One patient had significant bleeding in the upper gastrointestinal tract from a duodenal ulcer on the tenth postoperative day and underwent vagotomy and pyloroplasty. Two patients required pacemaker insertion before discharge for tachybradycardia syndrome.

Posthospitalization data. One patient died $6 \frac{1 / 2}{2}$ months after the operation with no postmortem examination. One patient was readmitted 7 months after the operation with positive blood cultures for Staphylococcus epidermidis and received intravenous antibiotic therapy for presumed endocarditis. No abnormality of the Freestyle valve was noted by
Table IV. Hemodynamic results determined by postoperative echocardiographic evaluation at 3 to 6 months (50 patients)

\begin{tabular}{ccc}
\hline Valve size & Gradient $(\mathrm{mm} \mathrm{Hg})$ & EOA $\left(\mathrm{cm}^{2}\right)$ \\
\hline $19 \mathrm{~mm}$ & $18.1(11.9-22.8)$ & $1.1(1.0-1.2)$ \\
$21 \mathrm{~mm}$ & $7.2(3.9-12.3)$ & $1.6(1.2-2.4)$ \\
$23 \mathrm{~mm}$ & $7.2(3.1-12.0)$ & $1.7(1.5-2.2)$ \\
$25 \mathrm{~mm}$ & $5.2(1.6-10.0)$ & $2.2(1.6-3.0)$ \\
$27 \mathrm{~mm}$ & $5.5(1.5-10.7)$ & $2.6(1.4-4.1)$ \\
\hline
\end{tabular}

Gradient, Mean aortic valve gradient; $E O A$, effective orifice area; range is given in parenthesis.

Table V. Hemodynamic results determined by echocardiographic evaluation at 1-year follow-up (nine patients)

\begin{tabular}{ccl}
\hline Valve size & Gradient $(\mathrm{mm} \mathrm{Hg})$ & EOA $\left(\mathrm{cm}^{2}\right)$ \\
\hline $19 \mathrm{~mm}$ & $10.0(9.0-11.0)$ & $1.5(1.4-1.6)$ \\
$21 \mathrm{~mm}$ & 6.0 & 1.6 \\
$23 \mathrm{~mm}$ & $7.5(5.0-10.0)$ & $1.8(1.7-1.8)$ \\
$25 \mathrm{~mm}$ & $8.0(7.0-9.0)$ & $1.6(1.4-1.9)$ \\
$27 \mathrm{~mm}$ & $4.0(2.0-6.0)$ & $2.7(2.1-3.3)$ \\
\hline
\end{tabular}

Gradient, Mean aortic valve gradient; $E O A$, effective orifice area; range is given in parenthesis.

echocardiographic evaluation and the patient has subsequently done well. Two patients required hospital admission and blood transfusion for gastrointestinal bleeding 1 and 4 months after the operation. All but four patients were in New York Heart Association class III or IV before the operation, and except for one patient, who is still in class III, all have improved to class I or II at the most recent follow-up visit.

Hemodynamic data. The mean aortic valve gradients and EOA for each valve size are listed in Table III. Table IV shows the mean gradients and the EOA determined by echocardiogram at the 3- to 6-month follow-up period. EOA increased and mean gradient decreased between the two studies. The nine patients who have undergone their 1-year follow-up echocardiograms (Table V) again show a further increase in the EOA and decrease in the mean aortic valve gradient. No patient had more than trace aortic insufficiency on any of the follow-up echocardiograms. Trace aortic valve insufficiency was noted in all patients receiving a $19 \mathrm{~mm}$ valve, one patient receiving a $21 \mathrm{~mm}$, three patients receiving a $25 \mathrm{~mm}$, and two patients receiving a 27 $\mathrm{mm}$ prosthesis.

The echocardiogram may overestimate the valve gradient. Two patients receiving a 19 and a $21 \mathrm{~mm}$ Freestyle valve underwent cardiac catheterization 
Table VI. Comparison of Freestyle valve with stented bioprothesis

\begin{tabular}{|c|c|c|c|c|c|}
\hline \multirow[b]{2}{*}{ Series } & \multicolumn{5}{|c|}{ Valve area $\left(\mathrm{cm}^{2}\right)$} \\
\hline & $19 \mathrm{~mm}$ & $21 \mathrm{~mm}$ & $23 \mathrm{~mm}$ & $25 \mathrm{~mm}$ & $27 \mathrm{~mm}$ \\
\hline \multicolumn{6}{|l|}{ Khan et al. ${ }^{11}$} \\
\hline Carpentier-Edwards* & 0.77 & 0.89 & 1.14 & 1.37 & 1.56 \\
\hline Hancock $^{*}$ & 0.85 & 1.11 & 1.59 & 1.5 & 1.38 \\
\hline \multicolumn{6}{|l|}{ David et al. ${ }^{12}$} \\
\hline Hancock II $\dagger$ & NA & 1.18 & 1.33 & 1.46 & 1.55 \\
\hline \multicolumn{6}{|l|}{ Freestyle } \\
\hline At discharge $\uparrow$ & 1.0 & 1.3 & 1.4 & 1.7 & 2.0 \\
\hline At 1 yeart & 1.5 & 1.6 & 1.8 & 1.6 & 2.7 \\
\hline
\end{tabular}

Carpentier-Edwards valve, manufactured by Baxter Healthcare Corp., Edwards Division, Santa Ana, Calif. Hancock II valve, manufactured by Medtronic Inc., Irvine, Calif.

*Gorlin area calculated from direct pressure measurements and thermodilution cardiac outputs in the operating room.

$\dagger$ Effective orifice areas obtained by Doppler echocardiography.

before hospital discharge. The measured valve gradients in these patients were less than $5 \mathrm{~mm} \mathrm{Hg}$, even though in one of these patients the discharge gradient by echocardiogram was $25 \mathrm{~mm} \mathrm{Hg}$.

\section{Discussion}

The first clinical implantation of a heterologous aortic valve was accomplished by Duran and Gunning in $1964 .{ }^{4}$ Binet and coworkers ${ }^{4}$ described the implantation of five aortic heterografts of both calf and pig origin preserved with 2-(ethyl-mercaptomercury) benzoxazol-5 sodium carbonate, and $\mathrm{O}^{\prime}$ Brien and Clareborough ${ }^{5}$ also used both porcine and calf aortic valves fixed with $4 \%$ buffered formalin as freehand aortic valve replacements in 23 patients in 1966. The initial results were excellent but the methods of tissue preservation used allowed early deterioration. It was not until the introduction of glutaraldehyde in 1968 by Alain Carpentier and his associates ${ }^{6}$ that bioprosthetic valves became reasonable alternatives to mechanical prostheses. After the commercialization of stented porcine bioprostheses by Hancock Laboratories in the early 1970 s, the further development of the more technically demanding stentless bioprostheses essentially came to a halt for the next 15 years. Recently, interest in glutaraldehyde-fixed nonstented porcine aortic valves has been renewed by the promise of improved forward flow hemodynamics and the potential for increased durability over the stented porcine bioprosthesis. Siebers and coworkers ${ }^{7}$ reported on the clinical use of a nonstented bioprosthesis in 1985. Bernhard and coworkers ${ }^{8}$ developed a scalloped, stentless valve supported with a flexible cloth-covered ring on the inflow side with the commissures covered with a thin layer of felt. This valve was clinically implanted for the first time in 1989 , and in 1991 it was reported to compare favorably with allograft valve replacement from the hemodynamic standpoint. ${ }^{9}$

David, Ropehan, and Butany ${ }^{10}$ began to implant an essentially stentless version of the Hancock II bioprosthesis in 1987 . This valve has fully scalloped sinuses and Dacron cloth-covered commissures and is sewn to the aortic root by means of a freehand technique. They compared 22 patients with the stentless valve to an equal number of patients undergoing aortic valve replacements with the Hancock II valve. Pressure gradients were significantly lower and the EOAs were considerably larger for the stentless bioprostheses compared with the stented valves.

The Freestyle aortic root design was based on a perceived need to have a readily available alternative to the human homograft. Because it is supplied as a complete aortic root it may be used for complete root replacement, implanted by a miniroot technique, or inserted freehand within the aortic root by scalloping the left and right coronary sinus portions or all three coronary sinuses as deemed necessary by the implanting surgeon. At present the Freestyle valve is not commercially available, but the price should be comparable with that of the stented modified-orifice Hancock bioprosthesis (personal communication from Medtronic Heart Valve, Irvine, Calif.).

Our data suggest that the hemodynamics of the stentless Freestyle valve are superior to those of stented bioprostheses (Table VI). The surgical technique to implant the Freestyle valve is somewhat more demanding, necessitating longer crossclamp times and attention to detail to achieve a completely 
competent valve. Because of the glutaraldehyde fixation and Dacron coverage of the muscle bar portion of the valve, it is more forgiving than an aortic homograft or a pulmonary autograft. Certainly any surgeon having experience with insertion of aortic homografts should be able to implant the Freestyle valve without difficulty.

Recently, significant discussion has been generated regarding the ideal method of implantation for homografts, autografts, and stentless bioprostheses. If durability of the valve indeed does depend on perfect coaptation of the valve leaflets, then implanting all three of these valves as complete roots may be the preferred method. Eleven of our patients do have trace aortic insufficiency, which has not progressed over the short-term follow-up period. Further long-term follow-up will be necessary to determine if this slight degree of insufficiency leads to decreased long-term durability of the valve.

We believe that several technical aspects are important when this stentless bioprosthesis is being implanted by means of the freehand technique. The valve should not be undersized, because so doing may create an outward displacement of the valve leaflets preventing them from coapting properly. We also believe that the Freestyle valve should not be used in large aortic roots, especially when there is a discrepancy between the diameter of the sinotubular junction and the patient's anulus. In this case implanting the Freestyle valve by a freehand technique will result in the commissures being pulled outward and prevent coaptation of the valve leaflets, resulting in aortic insufficiency. In these cases either a stented bioprosthesis should be used or the Freestyle valve should be implanted as a complete aortic root.

The increase in EOA and decrease in mean aortic valve gradients over time with the Freestyle valve has been observed by others using stentless porcine bioprostheses. The explanation for this phenomenon is not entirely clear, but it may be related to reabsorption of the blood or thrombus, which may collect between the native aortic wall and the bioprosthesis during the early phase after the operation. Certainly we have seen flow between the retained noncoronary sinus portion of the Freestyle valve and the patient's own aortic wall during the intraoperative transesophageal echo studies of these patients.

At present we believe that the Freestyle bioprosthesis is the valve of choice in patients requiring tissue valves and especially those with small anulus sizes. In addition, because the noncoronary sinus portion of the Freestyle valve is retained, this tissue can be used to enlarge the supravalvular aorta if necessary or to reinforce the often very thin aortic wall in the area of the aortotomy where it extends down into the noncoronary sinus portion of the native aorta. In summary, we believe that the stentless bioprosthesis is the valve of choice in the elderly patient with a small aortic root. Further long-term follow-up is required to determine the durability of this valve, although we believe it will last at least as long as the stented porcine bioprostheses presently available.

We thank Judy Fletcher, Carla Froelich, and Tim Dufrene for their help in the preparation of this manuscript.

\section{REFERENCES}

1. Morris DC, Wickliffe CW, King SB. Hemodynamic evaluation of the porcine heterograft aortic valve. Am J Cardiol 1976;37:157.

2. Schoen FJ, Hobson CE. Anatomic analysis of removed prosthetic heart valves: causes of failure of 33 mechanical valves and 58 bioprostheses, 1980-1983. Hum Pathol 1985;16:549-59.

3. Schoen FJ, Levy RJ. Bioprosthetic heart valve failure: pathology and pathogenesis. Cardiol Clin 1984;2:717.

4. Binet JP, Duran CB, Carpentier A, Langlois J. Heterologous aortic valve transplantation. Lancet 1965;2: 1275.

5. O'Brien MF, Clareborough JK. Heterograft aortic valve replacement. Lancet 1967;1:929-30.

6. Carpentier A, Lamaigre CG, Robert L, Carpentier S, Dubost C. Biological factors affecting long-term results of valvular heterografts. J THORAC CARDIOVASC SURG 1969;58:467-83.

7. Sievers HH, Lang PE, Bernhard A. Implantation of a xenographic stentless aortic bioprosthesis: first experience. Thorac Cardiovasc Surg 1985;33:225-6.

8. Bernhard A, Mahmoodi M, Sievers HH, Kraatz EG, Maurer I, Heitzer P. A "semi-supported" porcine xenograft-description and first clinical use. Thorac Cardiovasc Surg 1989;37:313-5.

9. Hausman B, Nellessen V, Hofig M, et al. Flexible aortic valve prosthesis: long-term functional results with porcine bioprostheses without mechanical commissure stent and aortic homografts. Z Cardiol 1991;80:51-8.

10. David TD, Ropchan GC, Butany JW. Aortic valve replacement with stentless porcine aortic bioprosthesis. J Thorac Cardiovasc Surg 1990;99:113-8.

11. Khan SS, Mitchell RS, Derby GC, Dyer GC, Miller DC. Differences in Hancock and Carpentier-Edwards porcine xenograft aortic valve hemodynamics. Circulation 1990;82(Suppl):IV117-24.

12. David TE, Armstrong S, Sun Z. Clinical and hemodynamic assessment of the Hancock II bioprosthesis. Ann Thorac Surg 1992;54:661-8. 


\section{Discussion}

Dr. Roger C. Millar (Salt Lake City, Utah). The search goes on for the ideal aortic substitute. It is exciting to use the homograft valve freehand techniques to implant the glutaraldehyde porcine tissue and to see if the durability of the aortic valve can be improved in this older group of patients. Ideally, this will provide proof in time to tell us if this valve will actually be better than the stented valves. We have had limited experience with this same valve, having been part of the study since January 1994 . We have not been anticoagulating our homografts and we have not anticoagulated our patients with this porcine stentless prosthesis either. I noted in your technique that you are including Teflon pledgets at all three commissures as part of the implantation procedure. We have found this glutaraldehydetreated tissue to be durable and have not thought it necessary to use prosthetic material, that is, Teflon felt. Do you think this is necessary? We worry that part of the reason to use this tissue valve is to avoid anticoagulation. You reported two cases of significant gastrointestinal bleeding and four cases of reexploration for pericardial effusions that possibly could be related to the anticoagulation process. Would you mind commenting on this?

Dr. Sintek. We started out not using anticoagulation in these patients and then three neurologic events occurred early in our series. At that point we decided that we should provide low-dose anticoagulation for 3 months. We have not been rigid about that. In very elderly patients, in their $80 \mathrm{~s}$, who are going to nursing homes or who do not have supervision at home, we continue the warfarin only for the duration of the hospital stay. There has been one transient ischemic attack in the follow-up period in one of those patients not receiving warfarin.

With regard to the pledgets, we have adopted the technique of Donald Ross, which is what we use for homografts and pulmonary autografts. We are comfortable with the technique. I agree that this valve is much more forgiving and much easier to work with than the homograft or certainly the autograft, but we still think that the resuspension in commissure is an important part of the operation. We have found that the tissue of the Freestyle aortic wall itself can be friable. When we did try in a couple of cases to not place the pledget, some of the stitches cut through the Freestyle valve. We are concerned that after the aorta is closed that may allow those commissures to pull down somewhat.

Dr. Millar. The data you gave on the EOA and the major gradients at discharge and 3 months later were interesting. You noted no change in the gradient across the valves from discharge to 3 months in the $19 \mathrm{~mm}$ size and virtually no change in the EOA. However, there was a decrease of nearly $50 \%$ in the gradient and an increase in the EOA in both the 21 and $23 \mathrm{~mm}$ sizes. Could you comment on these data and explain the reason?

Dr. Sintek. The increase in the EOA and the decrease in the aortic valve gradient over time have been observed by a couple of other groups implanting stentless valves. The explanation for the phenomenon is not entirely clear, and why there was a difference in the valve sizes I cannot explain. One possible explanation is that, over time, the blood or the thrombus that accumulates between the Freestyle valve and the patient's aortic wall is reabsorbed. During the intraoperative transesophageal echocardiograms of these patients we have seen flow between the retained noncoronary sinus portion of the Freestyle valve and the patient's aortic wall. Therefore there may be some continued flow or thrombus that forms there. Then, over the first 6 to 12 months after the operation, that may be reabsorbed and lead to a less bulky aortic wall above the level of the valve leaflets and therefore a better EOA and lower gradient.

Another possible explanation is that once the valve stenosis is relieved, the concentric hypertrophy in the left ventricular outflow tract may decrease somewhat. There may be some remodeling of the area right beneath the anulus. Many of these patients with aortic stenosis have a lot of muscle mass, and that may regress slightly with time and may provide for better flow characteristics.

Dr. Bradley J. Harlan (Sacramento, Calif.). The attractiveness of the hemodynamic advantages are of particular interest. I am intrigued that of your 60 patients only 20 had valves of $21 \mathrm{~mm}$ or less. We know that in this age group the durability is excellent with the stented bioprosthesis. What are your hypothetical advantages in the larger valve sizes?

Dr. Sintek. We agree with you completely. Part of the study protocol was that each center would implant 50 of these valves. Since fulfilling that obligation, we have been implanting these valves only in patients with 19 and $21 \mathrm{~mm}$ anulus sizes or in patients with a $23 \mathrm{~mm}$ anulus size who have a large body surface area. We see no hemodynamic advantage in the size 25 and $27 \mathrm{~mm}$ valves. There is a longer crossclamp time and that may lead to more perioperative complications. We agree with you that this valve is best reserved for the patient with a small anulus. 